Z. Klin. Chem. Klin. Biochem.

11. Jg. 1973, S. 224-232

\title{
Extrazelluläre cyclische Nucleotide: Vorkommen, Analytik und diagnostische Bedeutung
}

\author{
Von K.-D. Gerbitz und O. H. Wieland
}

Klinisch-chemisches Institut, Städt. Krankenbaus München-Scbwabing

(Eingegangen am 28. März 1973)

\author{
Herrn Professor Dr. Dr. Ernst Scbütte zum 65. Geburtstag gewidmet
}

\begin{abstract}
Cyclisches Adenosin-3',5'-monophosphat, dessen intrazelluläre Bedeutung als Signalübermittlex einer Vielzahl von Hormonen bekannt ist, sowie cyclisches Guanosin-3', $5^{\prime}$-monophosphat treten auch in den Extrazellulärraum über und können im Blutplasma, Urin und Liquor nachgewiesen werden. Die vorliegende Arbeit gibt eine Übersicht über das Gebiet dex extrazellulären cyclischen Nucleotide unter besonderer Berücksichtigung der bisher beschriebenen Testsysteme und der Möglichkeiten ihrer Anwendung in der Klinischen Chemie für Forschung und Diagnostik.
\end{abstract}

\section{Extracellular cyclic nucleotides: occurrence, analysis and diagnostic significance}

Cyclic-3',5'-AMP the well-known second messenger of many hormone actions, as well as cyclic-3', $5^{\prime}-\mathrm{GMP}$, do not only occur intracellularly but also in extracellular fluids such as blood plasma, cerebrospinal fluid, and urine. A review is given of the present knowledge of the extracellular cyclic nucleotides with special reference to analytical procedures and their application in clinical chemistry for research and diagnostic purposes.

Seit der Entdeckung des cyclischen Adenosin-3',5'monophosphat (Ado-3':5'-P) durch die Arbeitsgruppe um E. W. SutherLAND (1) und der in der Zwischenzeit immer deutlicher belegten Hypothese, die dieser Substanz eine Funktion als ",second messenger" einer Reihe von Proteohormonen und biogenen Aminen zuschreibt, hat 'die Erforschung des Mechanismus von'Hormonwirkungen in sprunghafter Weise neue Erkenntnisse gebracht. Für eine Reihe von Hormonen ist heute der in Abbildung 1 aufgezeigte Mechanismus anerkannt. Das vom ersten Sendboten - dem Hormon - an die Erfolgszelle zu übermittelnde Signal wird von einem spezifischen Empfänger - dem Hormonrezeptor - aufgenommen und über eine Überträgereinheit an ein membranständiges Enzymsystem - die Adenylcyclase - übermittelt, das die Uberführung von ATP in Ado-3': $5^{\prime}-\mathrm{P}$ katalysiert. Ein Anstieg der intrazellulären Konzentration an Ado-3' $: 5^{\prime}-\mathrm{P}$ bewirkt die Dissoziation einer Ado-3': $5^{\prime}$-P-abhängigen Proteinkinase in eine katalytische Einheit, die die eigentliche Phosphorylierungsreaktion katalysiert, und eine Bindungs- oder Regulatoreinheit, die spezifisch das cyclische Nucleotid bindet. Die unter dem Einfluß von Ado-3': $5^{\prime}-\mathrm{P}$ frei gewordene katalytische Einheit der Proteinkinase phos-

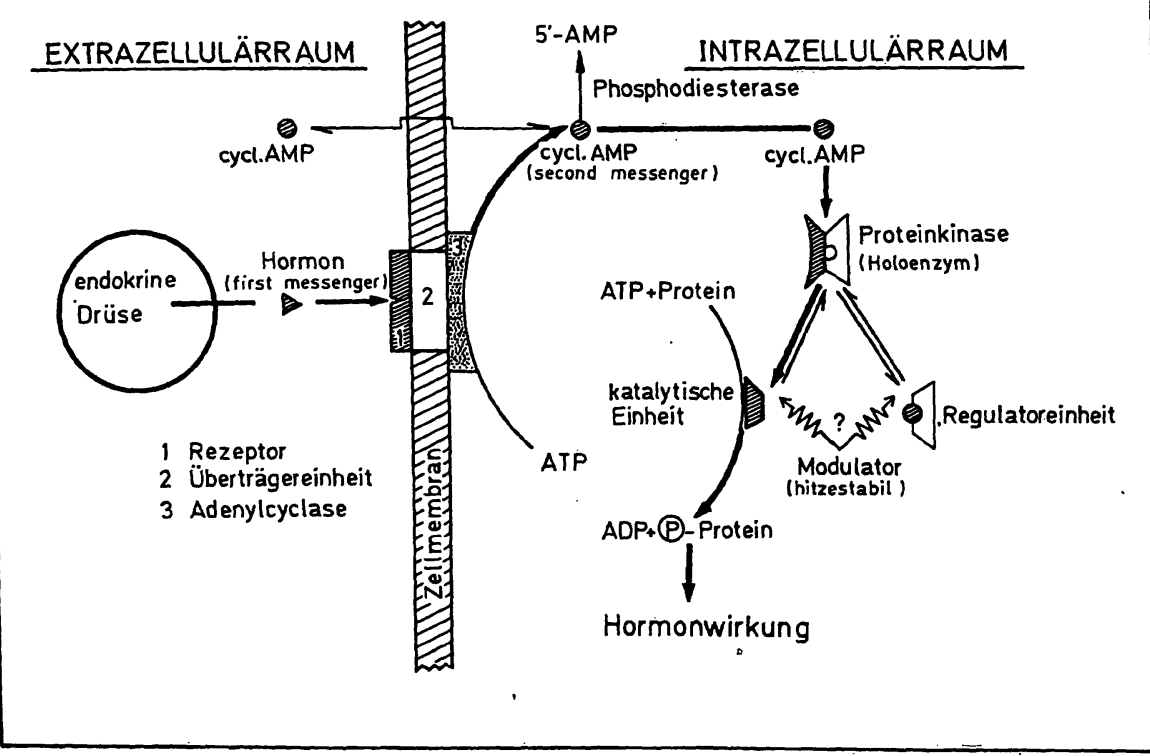

Abb. 1

Mechanismus durch Ado-3' : 5'-P vermittelter Hormonwirkungen 
phoryliert ein spezifisches Erfolgsprotein, dessen Eigenschaften dadurch gemäß dem Signal des jeweiligen Hormons verändert werden. Die Bindung des Ado$3^{\prime}: 5^{\prime}-\mathrm{P}$ an die Regulatoreinheit und die Aktivität der katalytischen Einheit werden nach neuesten Untersuchungen (2-7) auf bisher noch unbekannte Weise durch ein hitzestabiles "Modulatorprotein" beeinflußt. Am besten untersucht ist dieses System am Beispiel der Glycogen auf- und abbauenden Enzyme, Glycogen-Synthetase und Phosphorylase.

Der intrazelluläre Gehalt an Ado-3':5'-P wird geregelt durch das Syntheseenzym Adenylcyclase und das Abbauenzym Phosphodiesterase, das Ado-3':5'-P zu 5'-AMP abbaut. Weitere Möglichkeiten der Einflußnahme auf den intrazellulären Ado-3':5'-P-pool bestehen in der Bindung des Ado-3':5'-P an die Regulatoreinheit der Proteinkinase - so gebundenes Ado$3^{\prime}: 5^{\prime}-\mathrm{P}$ ist durch die Phosphodiesterase nicht angreifbar - , in anderen Abbauwegen des Ado-3': $5^{\prime}-P$, die zu bisher nicht identifizierbaren Abbauprodukten führen $(8,9)$, sowie im beschleunigten Efflux des Nucleotids in den Extrazellulärraum. Obwohl die starke Ladung des cyclischen Nucleotids einem schnellen Membrandurchtritt entgegensteht, ist zumindest für einige Gewebe ein erleichterter Transport diskutiert worden (10).

Seit es Butcher und SutherLand 1962 (11) erstmals gelang, Ado-3': 5'-P im Urin nachzuweisen, sind sowohl Ado-3': $5^{\prime}-\mathrm{P}$ als auch das zweite im Organismus vorkommende cyclische Nucleotid, cyclisches Guanosin$3^{\prime}: 5^{\prime}$-monophosphat (Guo-3':5'-P), in einer Reihe extrazellulärer Flüssigkeiten - Urin, Plasma, Liquor und Samenflüssigkeit - nachgewiesen worden $(7,12-35$, 103-105, Ref. 29). Diese bemerkenswerte Tatsache hat der klinisch-chemischen Forschung ein interessantes Aufgabengebiet eröffnet, das sich auf Zusammenhänge zwischen Extrazellulärspiegeln cyclischer Nucleotide und bestimmten Krankheitsbildern erstreckt. Nachdem zumindest für einige Gewebe eindeutige Beziehungen zwischen intra- und extrazellulären Konzentrationen der cyclischen Nucleotide bestehen, erșcheint die Möglichkeit gegeben, etwaige z. B. durch hormonelle Fehlregulation bedingte intrazelluläre Spiegelveränderungen an den extrazellulären Konzentrationen ablesen zu können. Schließlich bleibt die wichtige und noch offene Frage, ob den extrazellulär vorkommenden cyclischen Nucleotiden nicht eine besondere biologische Funktion zukommt $(29,99)$.

\section{Methoden zur Bestimmung der cyclischen Nucleotide}

Die cyclischen Nucleotide werden im Gewebe und im Extrazellulärraum in Konzentrationen zwischen $\mu \mathrm{mol} / 1$ und $\mathrm{nmol} / \mathrm{l}$ gefunden und liegen damit um mehrere Größenordnungen unter den Konzentrationen sowohl der anderen Purin - als auch der Pyrimidinnucleotide. Zur Erfassung derart niedriger Konzentrationen war die Ausarbeitung empfindlicher Testmethoden eine Vorbedingung. Die heute in der Literatur beschriebenen Testmethoden beruhen im wesentlichen auf folgenden Charakteristika:

1. Überführung von Ado-3':5'-P in andere Metabolite und dexen enzymatische Bestimmung

2. Aktivierung Nucleotid-abhängiger Proteinkinasen durch das jeweilige Nucleotid

3. Bindung an Antikörper oder spezifische Bindungsproteine

4. Chromatographische Methoden.

Úbersicht bei 1. c. $(36,37)$.

Bei der Wahl einer dieser Bestimmungsmethoden ist zu berücksichtigen, daß die Methode für die Fragestellung ausreichend sensitiv sein muß, daß sie einen möglichst großen Meßbereich überstreichen soll, daß der Arbeitsaufwand der Herstellung von Hilfsreagentien als auch der eigentlichen Messung nicht zu aufwendig ist, wozu als wichtigstes Kriterium gehört, daß die Methode spezifisch das zu messende Nucleotid bestimmt und keine Korrekturen des Ergebnisses erforderlich sind. Tabelle 1 gibt einen Vergleich verschiedener Methoden hinsichtlich ihrer Sensitivität und ihres Meßbereiches.

Die 1958 von Rall und Sutherland (38) entwickelte Methode der Messung von Ado-3': 5'-P durch Überführung der inaktiven Form der Phosphorylase in die aktive war mehrere Jahre Methode der Wahl. 1964 zeigte BRECKENRIDGE ein neues System auf. Die Sequenz führte dabei vom Ado-3':5'-P über eine gereinigte Phosphodiesterase zum 5'-AMP, von hier über die Reaktion der Adenylatkinase und der Pyruvatkinase

Tab. 1

Vergleich verschiedener Methoden zur Bestimmung von Ado-3': 5'-P

\begin{tabular}{|c|c|c|c|c|c|}
\hline Methode & Inkubationszeit & Inkubationsvolumen & $\begin{array}{l}\text { Meßbereich } \\
\text { pmol/Ansatz }\end{array}$ & Chromatographie & Markiertes Substrat \\
\hline STEINER et al. (21) & $4 \mathrm{~h}$ & $\begin{array}{l}150 \mu \mathrm{l} \\
600 \mu \mathrm{l}\end{array}$ & $\begin{array}{r}0,05-1,0 \\
0,5-100\end{array}$ & - & ${ }^{128} \mathrm{~J},{ }^{181} \mathrm{~J}$ \\
\hline BROOKER et al. (43) & $10 \mathrm{~min}$ & $35 \mu 1$ & $\begin{array}{l}5,0-200 \\
1,0-100\end{array}$ & $\begin{array}{l}+ \\
+\end{array}$ & ${ }^{3} \mathrm{H}-\mathrm{Ado}-3^{\prime}: 5^{\prime}-\mathrm{P}$ \\
\hline BROOKER (44) & & & $1,0-400$ & + & - \\
\hline Kuo (57) & $5 \mathrm{~min}$ & $186 \mu 1^{\circ}$ & $0,5-10,0$ & + & $\gamma$-s2P-ATP \\
\hline EBADI (41) & $6 \mathrm{~h}$ & & $0,05-$ & + & - \\
\hline GILMAN (62) & $60 \mathrm{~min}$ & $\begin{array}{r}50 \mu 1 \\
200 \mu l\end{array}$ & $\begin{array}{l}0,2-5,0 \\
1,0-20,0\end{array}$ & $\begin{array}{l}-(\cdot+) \\
-(+)\end{array}$ & ${ }^{2} \mathrm{H}-$ Ado-3' : 5'-P \\
\hline BRown et al. (63) & $90 \mathrm{~min}$ & $\begin{array}{r}50 \mu 1 \\
300 \mu 1\end{array}$ & $\begin{array}{l}0,1-5,0 \\
1,0-20,0\end{array}$ & $\begin{array}{l}-(+) \\
-(+)\end{array}$ & ${ }^{2} H-A d o-3 ': 5 '-P$ \\
\hline
\end{tabular}


zum ATP (39). ATP läßt sich dann auf mehrere Arten bestimmen: über die Reaktion der Hexokinase und der Pyruvatkinase (35), über die Markierung des ATP aus ${ }^{32} \mathrm{P}_{1}$ als Reaktion der Phosphoglyceratkinase und der Glyceraldehydphosphat-Dehydrogenase (40) oder als Substrat des Luciferin-Luciferase-Systems $(37,41,42)$. Diesen zuletzt genannten Methoden haftet, obwohl sie durch Verfeinerung Ado-3':5'-P noch in pmolMengen bestimmen können, der Nachteil an, daß das Nucleotid chromatographisch exakt von anderen $\mathrm{Nu}$ cleotiden abgetrennt werden muß und sie damit methodisch und zeitlich aufwendig sind.

Brooker (43) erweiterte 1968 das Spektrum der Meßmethoden um eine neue Variante, indem er vorschlug, im Sinne eines Isotopenverdünnungstestes als Substrat für die gereinigte Phosphodiesterase markiertes und unmarkiertes Ado-3': $5^{\prime}-\mathrm{P}$ anzubieten. $\mathrm{Um}$ in den niedrigen Konzentrationsbereich des Ado-3':5'-P im Gewebe zu kommen, war eine Phosphodiesterase mit sehr niedriger $\mathrm{K}_{m}$ für Ado-3':5'-P Voraussetzung. Ein solches Enzym wurde neben einer für Guo-3': $5^{\prime}-\mathrm{P}$ spezifischen Form im Rattenhirn gefunden (43). Dennoch ist diese Methode den meisten anderen an Sensitivität unterlegen. Die von Brooker $1970(37,44)$ veröffentlichte "high-pressure anion exchange chromatography" zur Messung von Ado-3': $5^{\prime}-\mathrm{P}$ und Guo- $3^{\prime}: 5^{\prime}-\mathrm{P}$ soll wegen ihres apparativen Aufwandes und den damit verbundenen Kosten nur am Rande erwähnt werden.

Der von STEINER et al. $(21,30,45,46)$ beschriebene hochsensitive Radioimmunoassay für Ado-3' : 5'-P, Guo$3^{\prime}: 5^{\prime}-\mathrm{P}$, Ino- $3^{\prime}: 5^{\prime}-\mathrm{P}$ und Urd-3': - $^{\prime}-\mathrm{P}$ beruht auf der Gewinnung von Antikörpern vom Kaninchen gegen die in $2^{\prime} \mathrm{O}-$ Position succinylierten und an Protein gebundenen cyclischen Nucleotide. Markiertes Substrat hoher spezifischer Aktivität wird durch Anhängung eines Tyrosinrestes an die succinylierten Derivate und nachfolgende ${ }^{131}$ Jod- oder ${ }^{125}$ Jod-Markierung gewonnen. Das Testprinzip ähnelt dem von YALOw und BERson für Insulin beschriebenen (47). Die Methode zeigt eine sehr hohe Sensitivität - bei entsprechender Reduzierung des Inkubationsvolumens können bis

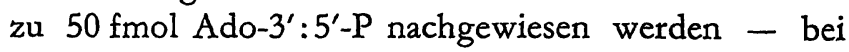
relativ weitgestrecktem Meßbereich. Ihre Spezifität (fehlende Kreuzreaktion mit anderen Nucleotiden) ist sehr gut. Bei Doppelmarkierung $\left({ }^{125} \mathrm{~J} /{ }^{131} \mathrm{~J}\right)$ lassen sich Ado-3': $5^{\prime}-\mathrm{P}$ und Guo-3':5'-P simultan bestimmen. Nachteilig sind die Aufwendigkeit der Antigen- und Antikörperherstellung und für Serienmessungen die relativ lange Inkubationszeit von $6 \mathrm{~h}$.

$\dot{D i e}$ Auffindung Ado-3' : 5'-P-abhängiger Proteinkinasen, zuerst in Skelettmuskel (48) und Leber (49), danach in einer Vielzahl unterschiedlicher Gewebe (50-54) und Guo-3': 5'-P-abhängiger Proteinkinasen bei Arthropoden $(55,56)$ ermöglichte neue Meßmethoden. Getreu dem in Abbildung 1 aufgezeigten Schema kann die Konzentration des cyclischen Nucleotides prinzipiell auf zweierlei Art bestimmt werden:
1. durch seine Eigenschaft, die Phosphorylierungsrate einer Proteinkinase konzentrationsabhängig zu stimulieren,

2. durch Bindung an die Regulatoreinheit einer Proteinkinase und Konkurrenz mit markiertem Ado-3': $5^{\prime}-\mathrm{P}$ um die Bindungsstelle im Sinne eines kompetitiven Isotopenverdünnungstestes..

Eine Reihe prinzipiell gleicher Methoden, die auf der Aktivierung der Kinasereaktion durch das jeweilige Nucleotid beruhen, ist in der Zwischenzeit publiziert worden $(57-60,102)$. Sie unterscheiden sich meist nur hinsichtlich der Präparation der verwendeten Proteinkinasen und der jeweiligen Proteinsubstrate.

Prinzip der Methode ist die Ubbertragung der markierten $\gamma$-Phosphatgruppe des ATP auf ein Akzeptorprotein, z. B. Histon, Protamin, Phosvitin oder Casein, durch eine von der Konzentration an Ado-3':5'-P oder Guo-3':5'-P-abhängige Proteinkinase. Das System ist hochspezifisch, hat aber methodische Nachteile: ${ }^{32} \mathrm{P}-$ markiertes ATP ist teuer und nicht lange haltbar. Neben der nötigen Proteinkinase muß auch das Substrat (z. B. Histon) selbst präpariert werden, da die kommerziellen Produkte häufig nicht genügend hohe Einbauraten zeigen (75). Limitiert ist die Methode durch die Tatsache, daß die gereinigten Proteinkinasepräparationen eine von exogenem Ado-3':5'-P-unabhängige Basisphosphorylierungsrate zeigen, die entweder durch eine Ado-3':5'-P-unabhängige Kinase oder durch endogen gebundenes Ado-3': $5^{\prime}-\mathrm{P}$ bedingt ist (36).

Walton und Garren (61), Gilman (62) und Brown et al. (63) veröffentlichten unabhängig voneinander 1970 Testmethoden für Ado-3' : $5^{\prime}-\mathrm{P}$, die auf der Bindung von markiertem cyclischen Nucleotid an die Regulatoreinheit einer Proteinkinase und dessen Verdrängung durch unmarkiertes Substrat als Standard oder unbekannte Probe im Sinne eines Isotopenverdünnungstestes beruht. Die Abtrennung des gebundenen ${ }^{3} \mathrm{H}-$ Ado-3':5'-P vom freien ${ }^{3} \mathrm{H}-\mathrm{Ado}-3^{\prime}: 5^{\prime}-\mathrm{P}$ geschieht durch Adsorption der Bindungseinheit an ein Milliporefilter $(61,62)$ oder durch Adsorption des freien Ado-3': $5^{\prime}-P$ an Aktivkohle (63). Filter bzw. im Überstand ' nach Zentrifugation verbleibendes, nicht an Kohle adsorbiertes Bindungsprotein können dann direkt in Szintillationsflüssigkeit ausgezählt werden. In der Zwischenzeit ist eine Reihe von Veränderungen der. Methodik beschrieben worden, die den störanfälligen Filtervorgang umgehen - durch Bindung der Proteinkinaseregulatoreinheit an Sepharose (66) -, die Inkubationsbedingungen verändern $(34,64-69,100)$ und neue Linearisierungsverfahren für die Eichkurven angeben $(64,65)$.

Durch Zugabe des eingangs erwähnten nach AppLEMAN et al. (4) präparierten „Modulatorproteins" läßt sich die Bindungskapazität der Regulatoreinheit erhöhen und vor allem die Gleichgewichtslage zwischen gebundenem und freiem Liganden stabilisieren (62).

Das zuletzt genannte, von uns übernommene und modifizierte System hat gegenüber den vorher beschriebenen Testmethoden entscheidende Vorteile: 
- die Anzahl der einzelnen Pipettierschritte ist gering - die Inkubationszeit ist mit $60-90 \mathrm{~min}$ nicht sehr lang

$-{ }^{3} \mathrm{H}-A d o-3^{\prime}: 5^{\prime}-\mathrm{P}$ ist billiger als die anderen markierten Substrate, hochmarkiert erhältlich und bei richtiger Handhabung auch haltbar

- die Präparation des Bindungsproteins aus Muskel; Herz oder Nebenniere vom Rind ist einfach und von hoher Ausbeute. Eine besondere Reinigung ist nicht unbedingt notwendig, manche Autoren arbeiten mit einer „high speed supernatant fraction" des homogenisierten Gewebes. Durch weitere Reinigung läßt sich die Sensitivität des Tests aber erhöhen. Das von uns aus Rinderherz in einem 6-Stufenarbeitsgang (51, 71) etwa $500 \mathrm{fach}$ angereicherte Bindungsprotein hat eine maximale Bindungskapazität für Ado- $3^{\prime}: 5^{\prime}-\mathrm{P}$ von $1,1 \mathrm{nmol} / \mathrm{mg}$ Protein. Material für etwa 60000 Tests wurde innerhalb zwei Tagen gewonnen. Eingefroren bei $-34^{\circ} \mathrm{C}$ ist das Bindungsprotein stabil und zeigt auch nach einem Jahr Lagerung keine Abnahme der Bindungskapazität.

- Ersetzt man den Filtervorgang durch Adsorption des freien ${ }^{3} \mathrm{H}$-Ado-3':5'-P an Kohle bzw. Dextran-Kohle, ergibt sich die Möglichkeit einer Automation sämtlicher Pipettiervorgänge. Manuell bewältigt eine eingearbeitete Assistentin in drei Stunden etwa 60 Proben mit Doppelwerten.

Die Auswertung der Ergebnisse erfolgt über eine Eichkurve, die mit primären Standards bekannter Konzentrationen an Ado-3': $5^{\prime}-\mathrm{P}$ erstellt ist. Die Linearisierung der Eichkurve ergibt sich nach GiLman (62) durch doppelt log. Auftragung, sofern im Sättigungsbereich an 'H-Ado-3':5'-P gemessen wird. Andere Auftragungsarten, die jeweils mit dem Grad der Sättigung variieren, sind die einfach reziproke Auftragung
$(64,65)$ und die „logit Transformation“ nach Rodbard et al. (72). Abbildung 2 zeigt den typischen Verlauf einiger mit unterschiedlicher Methodik gewonnener Eichkurven und deren Linearisierung.

Guo-3': $5^{\prime}-\mathrm{P}$, das zweite im Organismus vorkommende cyclische Nucleotid, kann nach MURAD et al. (73) ebenfalls nach dem oben beschriebenen Prinzip gemessen werden. Voraussetzung ist die Präparation einer Guo-3':5'-P-abhängigen Proteinkinase, wie sie hauptsächlich bei Arthropoden gefunden wird $(55,56,73)$. Ausgangsorgan ist meist der Schwanzmuskel von Hummer. Gilman und Murad haben die Kombination ihrer beiden Methoden zur Simultanmessung von Ado-3':5'-P und Guo-3':5'-P mit Doppelmarkierung beschrieben (74). Aufgrund der Spezifität beider Kinasen bzw. der Bindungsproteine für das jeweilige Nucleotid interferieren die beiden cyclischen Nucleotide nicht gegenseitig, wenn sie in einem Inkubationssystem bestimmt werden (74). Auch andere Nucleotide interferieren im Test erst in sehr hohen, meist weit über den $z \mathrm{u}$ erwartenden Spiegeln liegenden Konzentrationen $(62,64,65,74,75)$.

\section{Probenauf bereitung zur Bestimmung der Extra- zellulärkonzentrationen cyclischer Nucleotide}

Die folgenden Angaben über die Aufarbeitung von Urin und Plasmaproben beziehen sich auf Messungen der cyclischen Nucleotide mit der Proteinbindungsmethode $(62-64,73,74)$.

Urinproben brauchen nach übereinstimmenden Angaben in der Literatur nicht vorbehandelt oder gereinigt $\mathrm{zu}$ werden, auch eine Zugabe von PhosphodiesteraseHemmstoffen (Methylxanthine, EDTA) ist nicht nötig, sofern die Proben möglichst bald tiefgekühlt werden. Wir konnten bei $24 \mathrm{~h}$ dauernder Lagerung bei Raum-

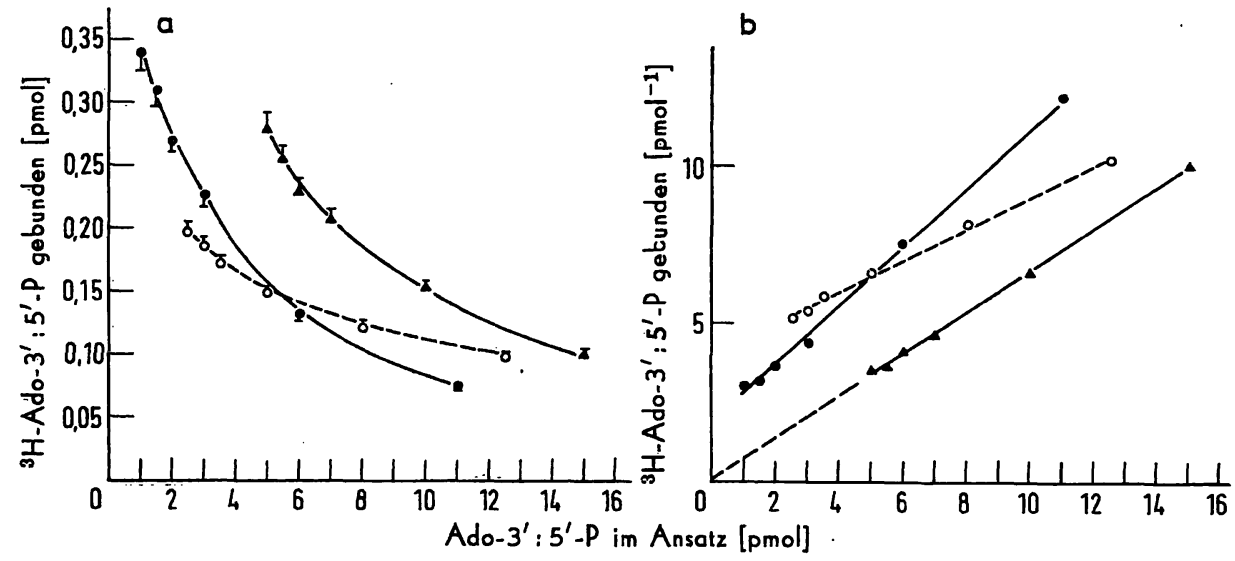

Abb. 2

Typische Ado-3': 5'-P Standardkurven unterschiedlicher Meßmethodik (a) und deren Linearisierung (b)

Bedingungen: - - nach GILMAN (62), Inkubationsvolumen $200 \mu 1,1$ pmol ${ }^{3} \mathrm{H}-\mathrm{Ado}-3^{\prime}: 5^{\prime}-\mathrm{P}$ (37000 dpm), 0,78 $\mu \mathrm{g}$ Bindungsprotein, $40 \mathrm{mmol} / 1$ Natriumacetatpuffer,pH 4,0

- nach GILMAN (62), Inkubationsvolumen $200 \mu \mathrm{l}, 5 \mathrm{pmol}{ }^{2} \mathrm{H}-\mathrm{Ado}-3^{\prime}: 5^{\prime}-\mathrm{P}$ (185000 dpm), $0,31 \mu \mathrm{g}$ Bindungsprotein, $40 \mathrm{mmol} / \mathrm{l} \mathrm{Natriumacetat-}$ puffer, $\mathrm{pH} 4,0$

Inkubationszeit 75 min bei $0^{\circ} \mathrm{C}$ O-O nach BRown et al. (63), Inkubationsvolumen $200 \mu \mathrm{l}, 2,5$ pmol ${ }^{\prime} \mathrm{H}-\mathrm{Ado}-3^{\prime}: 5^{\prime}-\mathrm{P}$ (92500 dpmi), $0,78 \mu \mathrm{g}$ Bindungsprotein, $40 \mathrm{mmol} / \mathrm{l}$ Natrium-
acetatpuffer, $\mathrm{pH} 4,0^{\circ}$

Inkubationszeit $75 \mathrm{~min}$ bei $0^{\circ} \mathrm{C}$

Die Trennung des gebundenen vom freien Ado-3':5'-P erfolgte beim GilmAN-Test durch Filtration an Milliporefilter (HAWP 02400) und

nachfolgendem Waschen mit $3 \mathrm{ml} 20 \mathrm{mmol} / 1$ Kaliumphosphatpuffer, pH 6,2 , bei dem in Anlehnung an BRowN et al. (63) durchgeführten

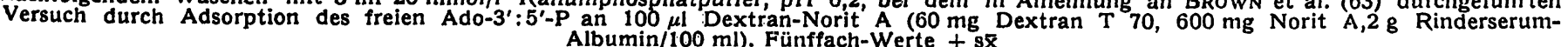

Die Linearisierung der Eichkurven erfolgte durch einen einfach reziproken Plot nach $(64,65)$ 
temperatur keine Abnahme der Ado-3':5'-P-Spiegel gegenüber sofort tiefgefrorenen Proben feststellen (75). Ado-3': $5^{\prime}-\mathrm{P}$ und Guo-3':5'-P lassen sich direkt in den entsprechend verdünnten Proben bestimmen.

Heparinisiertes Vollblut sollte unmittelbar nach Abnahme mit einem Phosphodiesterase-Hemmstoff (Theophyllin, EDTA) versehen und nach Zentrifugation das Plasma, sofern es nicht sofort weiterverarbeitet wird, eingefroren werden. Über die Notwendigkeit einer weitergehenden Reinigung von Plasmaproben sind die Angaben in der Literatur widersprüchlich. Während einige Autoren Ado-3': $5^{\prime}-\mathrm{P}$ direkt in deproteinisierten Plasmaextrakten bestimmen konnten $(34,76)$, halten wir in Übereinstimmung mit anderen $(33,77)$ chromatographische Reinigungsverfahren für nötig, da auch die mit den üblichen Deproteinisierungsverfahren (Trichloressigsäure, Perchlorsäure, Äthanol) behandelten Plasmen Faktoren enthalten, die die Bindungsmethode direkt stören (75). Das gilt in analoger Weise für Messungen der cyclischen Nucleotide in verschiedenen Geweben $(65,100)$. Die chromatographische Reinigung und Abtrennung der cyclischen Nucleotide von anderen Nucleotiden kann an Kationenaustauschern
(Dowex $50 \mathrm{~W}-\mathrm{X} 4$ ) (97) oder Aluminiumoxid $(95,96)$ vorgenommen werden. Nach unseren Erfahrungen kann gesagt werden, daß die Proteinbindungsmethode, in welcher Variation sie auch ausgeführt wird, an die verschiedenen Gewebe bzw. Extrazellulärflüssigkeiten, in denen die cyclischen Nucleotide bestimmt werden sollen, speziell adaptiert werden muß. Eingehende Kontrollen (Leerversuche, Wiederfindung und Andauung durch Phosphodiesterase) sind neben einer genauen Standardisierung der Methode als Kontrolle unerläßlich.

\section{Extrazelluläre Konzentrationen cyclischer Nucleotide}

Beide cyclische Nucleotide sind im Plasma nicht an Protein gebunden nachweisbar (14), entstammen nur zum unwesentlichen Anteil den korpuskulären Bestandteilen dès Blutes und werden glomerulär filtriert im Urin ausgeschieden. Zwischen intrazellulärer Synthese der Nucleotide, Durchtritt durch die.Zellmembran, Abbau und Ausscheidung besteht ein Fließgleichgewicht. Die Plasmaproduktionsraten liegen zwischen 9-17 $\mathrm{nmol} / \mathrm{min}$ für Ado-3' : $5^{\prime}-\mathrm{P}$ und $7-13 \mathrm{nmol} / \mathrm{min}$

Tab. 2

Extrazelluläre Konzentrationen cyclischer Nucleotide beim Menschen

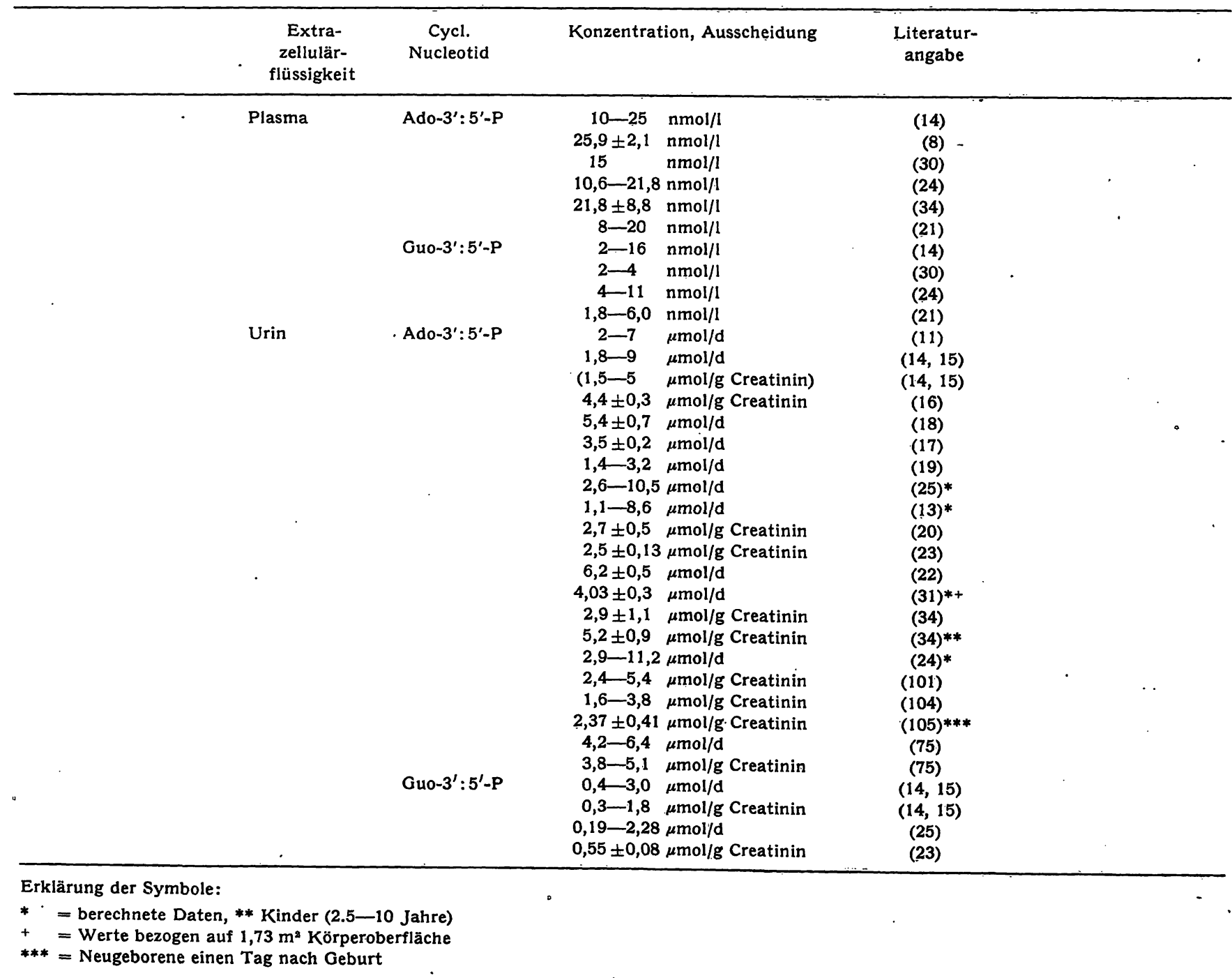


für Guo-3': $5^{\prime}-\mathrm{P}$ (14), die Halbwertszeit für Ado-3' $: 5^{\prime}-\mathrm{P}$ beträgt etwa 30 min. Rund $20 \%$ des Plasma-Ado-3' $: 5^{\prime}-\mathrm{P}$ und $-G u o-3^{\prime}: 5^{\prime}-P$ werden glomerulär filtriert von der Niere ausgeschieden, die Clearance des verbleibenden Restes aus dem Plasma geschieht auf andere weitgehend noch unbekannte Weise, wobei zumindest für einen Teil ein Membrandurchtritt in den Intrazellulärraum, z. B. der Erythrocyten, diskutiert werden muß. $\mathrm{Ob}$ dieser Annahme eine physiologische Bedeutung zugrunde liegt, ist noch unklar (99). Während der Gesamtteil des im Urin erscheinenden Guo-3': $5^{\prime}-\mathrm{P}$ glomerulär durch die Niere filtriert wird, stammt ein variierender Anteil des Urin-Ado-3': $5^{\prime}-\mathrm{P}$ aus der Niere selbst als Ausdruck der in den proximalen Nierentubuli (13) lokalisierten Parathormon-sensitiven Adenylcyclase $(13-15,26,40,82)$. Dieser Parathormon-bedingte Anteil scheint individuell konstant zu sein, variiert aber von Individuum $z u$ Individuum, so da $B$ eine eindeutige Korrelation zwischen Plasma-Ado-3': $5^{\prime}-\mathrm{P}$ Spiegel und Urinausscheidung an Ado- $3^{\prime}: 5^{\prime}-\mathrm{P}$ im Kollektiv nicht gegeben erscheint. Für die Ado-3': $5^{\prime}-\mathrm{P}$ Ausscheidung ist daher neben der Angabe bezogen auf die Ausscheidung pro Zeiteinheit (24-Stundensammelurin) auch die Beziehung zur Creatininausscheidung notwendig. Angaben über die in der Literatur beschriebenen und mit unterschiedlichen Methoden gemessenen Extrazellulärspiegel der cyclischen Nucleotide sind in Tabelle 2 zusammengestellt. Eine Übersicht über die Literatur bis 1970 gibt (29).

\section{Veränderungen der Extrazellulärspiegel der cycli- schen Nucleotide durch exogene Hormongaben}

1967 beschrieben Chase und Aurbach (78) bei der Ratte die Stimulierung der Ado-3':5'-P-Ausscheidung durch Gabe von Parathormon. Die Befunde sind mittlerweile auch für den Menschen bestätigt (13, 15, $16,17,25)$. Patathormongabe bedingt einen dosisabhängigen Anstieg der Ado-3' : 5'-P-Ausscheidung und des Plasma-Ado-3':5'-P-Spiegels $(15,25)$ sowie der

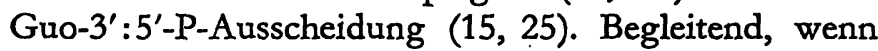
auch verzögert, kommt es zum Anstieg der Phosphatausscheidung. Calcium-Infusionen drücken den Parathormon-bedingten Anteil des Urin-Ado-3': $5^{\prime}-\mathrm{P}$, wenn auch das Clearance-Verhältnis Ado-3': $5^{\prime}$-P/Inulin nicht den Wert 1 erreicht. Dies macht es wahrscheinlich, daß neben Parathormon die Niere noch Erfolgsorgan anderer die Ado-3' : 5'-P-Ausscheidung stimulierender Hormone ist (15). Uber den Anteil des Adiuretins (Vasopressin), dessen Funktion in einer Ado-3':5'-P-vermittelten Steigerung der Wasserpermeabilität der distalen Tubuli und der Sammelrohre (79) besteht, an der Ado-3': $5^{\prime}$-P-Ausscheidung sind die Angaben in der Literatur widersprüchlich. Während von einzelnen Autoren $(17,20)$ deutliche Anstiege der Ado-3':5'-PAusscheidung nach Adiuretin angegeben wurden - wenn auch teilweise nur bei sehr hoher Dosierung konnten diese Befunde von anderen nicht bestätigt werden $(13,15)$.
Insulin, dessen senkende Wirkung auf den intrazellulären Spiegel an Ado-3':5'-P in einigen Organen beschrieben ist (3), hat keinen Einfluß auf die PlasmaKonzentrationen des cyclischen Nucleotids bzw. seine Ausscheidung beim Menschen (14), steigert dagegen die Ado-3': $5^{\prime}$-P-Spiegel in Leber, Muskel und Blut beim Hahn (28). Glucagon führt zu einem starken Anstieg des Plasma-Ado- $3^{\prime}: 5^{\prime}-\mathrm{P}$ - Quelle ist hier die Leber (14) - und des Urin-Ado-3': $5^{\prime}-\mathrm{P}(14,25)$. Uber das Verhalten von Guo-3' : 5'-P nach Glucagongabe sind die Angaben in der Literatur unterschiedlich. BROADus et al. (14) fanden keine Änderung des Plasma-Guo-3' : 5' P-Spiegels und der Guo-3':5'-P-Ausscheidung nach Glucagongabe, WiLLIAMs et al. konnten dagegen mit gleich hohen Glucagongaben eine deutliche Erhöhung

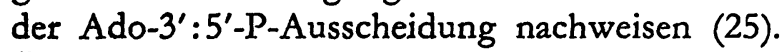

Ähnlich widersprüchlich sind die Angaben über das Verhalten von Guo-3': $5^{\prime}-\mathrm{P}$ nach Gabe von Catecholaminen. Während BALL et al. (24) in der Reihe der Catecholamine mit Zunahme der $\alpha$-adrenergen Komponente eine Erhöhung von Plasma- und Urin-Guo$3^{\prime}: 5^{\prime}-P$ fanden, die sich durch gleichzeitige Gabe eines $\beta$-Blockers verstärken, durch Gabe eines $\alpha$-Blockers hemmen ließ, wogegen das $\beta$-adrenerge Isoproterenol ohne Wirkung war, fanden Williams et al. (25) nach Gabe von Isoproterenol eine stärkere Steigerung der Ausscheidung von Guo-3': $5^{\prime}-\mathrm{P}$ als von Ado-3':5'-P. Epinephrin, mit oder ohne $\alpha$ - oder $\beta$-Blocker gegeben, hat nach WILliams et al. (25) keinen signifikanten Effekt auf die Ado-3' : 5'-P-Ausscheidung. Dagegen fanden BALL et al. (24) deutliche Anstiege der Plasma-Ado-3' : 5'-P-Spiegel, wie auch der Ausscheidung nach Gabe von Epinephrin, die sich aufgrund der möglichen Blockade durch einen $\beta$-Blocker der $\beta$-adrenergen Komponente des Hormons zuordnen ließ. Ob der $\alpha$-adrenerge Stimulus eine Direktwirkung auf die Guo-3':5'-P-Plasmakonzentration und Urinausscheidung darstellt, oder ob Sekundärmechanismen mitspielen, ist noch unklar $(24,70,80)$.

ACTH hat keinen Effekt auf die Ado-3':5'-P-Ausscheidung der Ratte (78) und des Menschen (25), obwohl es nach Gabe von ACTH zu extremen Anstiegen der intrazellulären Konzentrationen an Ado$3^{\prime}: 5^{\prime}-\mathrm{P}$ in der Nebenniere kommt. Corticosteroide und Thyroxin, die an der hypophysektomierten bzw. thyreoparathyreoidektomierten Ratte den Abfall der Ado$3^{\prime}: 5^{\prime}-\mathrm{P}$ und Guo-3':5'-P-Ausscheidung in gewissen Fällen verhindern (27), haben keine oder nur geringgradige Effekte beim Menschen (25).

Angaben über die Extrazellulärspiegel der cyclischen Nucleotide nach Prostaglandingaben sind uns nicht bekannt. Prostaglandin $\mathrm{E}_{1}$ hemmt die Parathormonsensitive Adenylcyclase und den Anstieg des intrazellulären Ado-3' : 5'-P nach Gabe von Parathormon (106). Generell kann gesagt werden, daß durch unterschiedliche Hormone intrazellulär an den jeweiligen Erfolgsorganen bedingte Konzentrationsänderungen der cyclischen Nucleotide sich nicht in jedem Fall in Änderungen auch der Extrazellulärkonzentrationen zu äußern brauchen. Von Gewebe zu Gewebe unter- 
schiedliche Flux- und Transportraten und die unterschiedliche Bindung der Nucleotide an verschiedene Membranstrukturen scheinen eine wesentliche Rolle zu spielen (81).

\section{Pathophysiologie}

Obwohl im Tierexperiment und auch beim Menschen Beeinflussungen der Extrazellulärspiegel der cyclischen Nucleotide durch verschiedene hormonelle Faktoren beschrieben sind, gibt es in der Literatur relativ wenige Berichte über Untersuchungen der Nucleotidspiegel bei pathologischen Zuständen. CHASE et al. (13) untersuchten Patienten mit verschiedenen Krankheitsbildern des Hyper- und Hypoparathyreoidismus. Idiopathische und postoperative Fälle von Hypoparathyreoidismus zeigten eine unter der Norm liegende Ado-3':5'-PAusscheidung, die durch Gabe von Parathormon erhöht werden konnte, wogegen beim Pseudohypoparathyreoidismus nach Gabe von Parathormon kein Anstieg der Ado-3':5'-P-Ausscheidung gefunden wurde. In diesen Fällen von Pseudohypoparathyreoidismus ist das radioimmunologisch im Plasma nachweisbare Parathormon über die Norm erhöht und kann durch Ca-Infusionen gesenkt werden. Hier scheint die Ursache in einer verminderten Ansprechbarkeit der Erfolgsorgane Niere und Knochen auf Parathormon zu liegen, obwohl die corticale Adenylcyclase in vitro durch Parathormon stimulierbar ist (82). Hyperparathyreoidismus (Adenom, Carcinom) bedingt. eine erhöhte Ausscheidung an Ado-3': $5^{\prime}-\mathrm{P}(15,16,17,22,23,101)$, wogegen die Angaben über Veränderungen der Guo$3^{\prime}: 5^{\prime}$-P-Ausscheidung nicht eindeutig sind. Nicht durch Parathormon bedingte Hypercalcämien (Ursache: Carcinom oder Sarcoidose) zeigen dagegen keine Änderungen der Ado-3':5'-P- oder Guo-3':5'-P-Ausscheidung im Vergleich zu Gesunden. MURAD und PAK halten daher die gesteigerte Ado-3':5'-P-Ausscheidung bei einer Parathormon-bedingten Hypercalcämie für ein gutes Differentialdiagnostikum (23).

In Analogie zum Pseudohypoparathyreoidismus fanden FichMan und Brooker (20) bei zwei Patienten mit nephrogenem Diabetes insipidus keinen Anstieg der Ado-3':5'-P-Ausscheidung nach Gabe von Adiuretin, wogegen bei Gesunden und in fünf Fällen von Primärem Diabetes insipidus Adiuretin-Gabe die Ado$3^{\prime}: 5^{\prime}$-P-Ausscheidung steigerte.

Sulfonylharnstoffe, deren möglicherweise durch Hemmung der Phosphodiesterase bedingter (86) antidiuretischer Effekt seit längerem bekannt ist $(83-85$, 98) haben beim nephrogenen Diabetes insipidus keinen Einfluß auf die Ausscheidung von Ado-3': $5^{\prime}-\mathrm{P}$ (20). Erniedrigte Ado-3':5'-P-Ausscheidungstaten finden sich beim cyclothymen Formenkreis während der depressiven Phase $(18,19)$, steigen dagegen kurz vor Umschlag in die manische Phase an (32). Ob diese
Tatsache Ausdruck einer erhöhten Catecholaminausschüttung ist, ist noch nicht ganz geklärt (32). Behandlung mit L-Dopa steigert die Ado-3':5'-P-Ausscheidung (cit. nach 32), ebenso Behandlung mit tricyclischen Antidepressiva (19), die starke Hemmstoffe der Phosphodiesterase sind. Im Tierexperiment konnte an Hirnschnitten der Einfluß einer ganzen Reihe von zentral angreifenden Substanzen auf die cerebralen Ado-3': $5^{\prime}$-P-Spiegel nachgewiesen werden, dazu gehören Antidepressiva, Neurotransmitter und Tranquilizer (87-89, 93).

Elektroschock führt zum Anstieg der cerebralen Ado-3': ' $^{\prime}-\mathrm{P}$ - und Guo-3' $: 5^{\prime}$-P-Spiegel im Tierexperiment $(90,91)$, beim Menschen zum Anstieg der Ado-3':5'-PAusscheidung im Urin (92). Durch Gabe von Probenecid kommt es beim Menschen zum Anstieg von Ado$3^{\prime}: 5^{\prime}-\mathrm{P}$ im. Liquor der möglicherweise durch eine Blockade auf Höhe der Blut/Liquorschranke bedingt ist $(10,33)$, bei absinkender Ado- $3^{\prime}: 5^{\prime}-\mathbf{P}$-Ausscheidung und unverändertem Plasma-Ado-3' : 5'-P-Spiegel (33). Unter der Norm liegende Ado-3':5'-P-Ausscheidungsraten zeigen chron. Asthmatiker $(31,94)$. In diesen Fällen konnte die verminderte Ausscheidung des cyclischen Nucleotids durch Glucagongabe gesteigert werden, im Gegensatz zu gesunden Versuchspersonen jedoch nicht durch Gabe von Epinephrin (31). BERNSTEIN et al. schließen aus diesem Befund auf einen Defekt der Epinephrin-stimulierbaren Adenylcyclase bei Asthmatikern und halten es für möglich, da $\beta$ hier ein grundsätzliches ätiologisches Moment dieses Leidens gegeben ist.

Gemessen an der Erkenntnisfülle, die die Entdeckung der cyclischen Nucleotide für das Verständnis der Wirkung von Hormonen erbracht hat, muß unser Wissen über die Bedeutung und das Verhalten dieser Substanzen, nachdem sie das Zellinnere verlassen haben, noch als bescheiden angesehen werden. Ein Vergleich der von den einzelnen Autoren angegebenen Konzentrationsänderungen der cyclischen Nucleotide bei verschiedenen Krankheitsbildern macht deutlich, $\mathrm{da} ß$ sich in vielen Fällen für signifikant gehaltene Spiegeländerungen innerhalb eines Konzentrationsbereiches abspielen, der von anderen Autoren noch als Normbereich angesehen wird. Mit anderen Worten, genaue Aussagen werden sich erst machen lassen, wenn positive Korrelationen der Syntheseraten der cyclischen Nucleotide zu Alter, Geschlecht, Gewicht, Größe, Körperoberfläche, Muskelmasse und Stoffwechselzustand an genügend großen statistisch gesicherten Kollektiven erstellt sind.

\section{Danksagung}

Die Arbeit wurde mit Unterstützung durch das Bundesministerium für Forschung und Technologie durchgeführt. Fräulein BiRTHE LüBBEN danken wir für ausgezeichnete technische Assistenz. 


\section{Literatur}

1. Rali, T. W. \& Suthibrland, E. W. (1958), J. Biol. Chem. 232, 1065. - 2. Gonzales, C.: M. S. Thesis, University of Washington 1962. - 3. Posner, J. B., Stera, R. \& Krebs, E. G. (1965), J. Biol. Chem. 240, 982-985. - 4. Appleman, M. M., Birnbaumer, L. \& Torres, H. N. (1966), Arch. Biochem. Biophys. 116, 39. - 5. Walsh, D. A., Ashby, C. D., Gonzales, C., Calkins, D., Fischer, E. H. \& KREBs, E. G. (1971), J. Biol. Chem. 246, 1977-1985. - 6. AshBy, C. D. \& Walsh, D. A. (1972), J. Biol. Chem. 247, 6637-6642. - 7. Donnelly, T. E. jr., Kuo, I. F., ReYes, P. L., Liu, Y.-P. \& Greengard, P. (1973), J. Biol. Chem. 248, 190. - 8. Murad, F., Rak, T. W. \& VaughaN, M. (1969), Biochim. Biophys. Acta 192, 430. - 9. Rosison, G. A., Butcher, R. W. \& Sutherland, E. W. (1971), Cyclic AMP. Academic Press, New York-London. - 10. Davoren, P. R. \& Sutherland, E.W. (1963), J. Biol. Chem. 238, 3009.-11. Butcher, R. W. \& Sutherland E.W. (1962), J. Biol. Chem. 237, 1244. 12. Takahashi, K., Kamimura, M., Shinko, T. \& Tsuji, S. (1966), Lancet II, 967. - 13. Chase, L. R., Melson, G. L. \& AurbaCli, G. D. (1969), J. Clin. Invest. 48, 1832-1844. - 14. Broadus, A. E., Kaminsky, N. I., Hardman, J. G. Sutherland, E. W. \& LidDle, G. W. (1970), J. Clin. Invest. 49, 2222-2236. - 15. Kaminsky, N. I., Broadus, A. E., Hardman, J. G., Jones, D. J., Ball, J. H., Sutherland, E. W. \& Liddde, G. W. (1970), J. Clin. Invest. 49, 2387-2393. - 16. Estep, H., Fratkin, M., Moser, A. \& Robinson, F. (1970), Clin. Res. 18, 358. - 17. Taylor, A. L., Davis, B. B., Paulson, L. G., Josimovich, J. B. \& Mrntz, D. H. (1970), J. Clin. Endocrinol. Metab. 30, 316-324. 18. Paul, M. I., Ditzion, B. R., Pauk, G. L. \& Janowsky, D. S. (1970), Amer. J. Psychiat. 126, 1493. - 19. Abdullah, Y. H. \& Hamadah, K. (1970), Lancet I, 378-381. - 20. Fichman, M. P. \& Brooker, G. (1972), J. Clin. Endocrinol. Metab. 35, 35-47. - 21. Steiner, A. L., Pagliara, A. S., Chase, L. R. \& KipNIS, D. M. (1972), J. Biol. Chem. 247, 1114-1120. - 22. Dohan, P. H., Yamashita, K., Larsen, P. R., Davis, B., Deftos, L. \& Freld, J. B. (1972), J. Clin. Endocrinol. Metab. 35, 775-784. 23. Murad, F. \& PaK, C. Y. C. (1972), N. Engl. J. Med. 286, 1382-1387. - 24. Ball, J. H., Kaminsky, N. I., Hardman, J. G., Broadus, A. E., Sutherland, E. W. \& Liddle, G. W. (1972), J. Clin. Invest. 51, 2124-2129. - 25. WILliams, R. H., Barish, J. \& Ensinck, J. W. (1972), Proc. Soc. Exp. Biol. Med. 139, 447-454. - 26. Chase, L. R. \& Aurbach, G. D. (1967), Proc. Nat. Acad. Sci. USA 58, 518-525. - 27. Hardman, J. G., Davis, J. W. \& Sutherland, E. W. (1969), J. Biol. Chem. 244, 6354-6362. - 28. Frohlich, A. A. \& Marquardt, R. R. (1972), Biochim. Biophys. Acta 286, 396-405. - 29. Broadus, A. E., Hardman, J. G., Kaminsky, N. I., Ball, J. H., Sutherland, E. W. \& Liddle, G. W. (1971), Ann. N. Y. Acad. Sci. 185, 50-66. - 30. Steiner, A. L., Parker, C. W. \& Kipnis, D. M. (1970), Advan. Biochem. Psychopharmacol. (Costa, E. \& Greengard, P., Eds.) vol. 1, Raven Press, New York, N. Y. - 31. Bernstein, R. A., Linarelit, L., FACKTOR, M. A., Friday, G. A., Drash, A. L. \& Fireman, P. (1972), J. Lab. Clin. Med. 80, 772-779. - 32. Paul, M. I., Cramer, H. \& BunNey, W. E. jr. (1971), Science 171, 300-303. - 33. Cramer, H., NG, L. K. J. \& ChASE, T. N. (1972), J. Neurochem. 19, 1601. 34. Tsang, C. P. W., Lehotay, D. C. \& Murphy, B. E. P. (1972), J. Clin. Endocrinol. Metab. 35, 809-817. - 35. Goldberg, N. D., Larner, J., Sasko, H. \& O'Toole, A. G. (1969), Anal. Biochem. 28, 523-544. - 36. BRECKENRIDGE, B. MCL. (1971), Ann. N. Y. Acad. Sci. 185, 10-17. - 37. GreEngard, P. \& Robison, G. A. (1972), Advan. Cycl. Nucleotide Research, Vol. 1, 2, Raven Press, New York. - 38. RalL, T. W. \& Sutherland, E. W. (1958), J. Biol. Chem. 232, 1065. 39. Breckenridge, B. McL. (1964), Proc. Nat. Acad. Sci. USA. 52, 1580. - 40. Aurbach, G. D. \& Houston, B. A. (1968), J. Biol. Chem. 243, 5935. - 41. Ebadi, M. S., Werss, B. \& Costa, E. (1970), Science 170, 188-190. - 42. Johnson, R. A., HARDman, J. G., Broadus, A. E. \& Sutherland, E. W. (1970), Anal. Biochem. 35, 91. - 43. Brooker, G., Thomas, L. J. \& AppleMAN, M. M. (1968), Biochemistry 7, 4177-4182. - 44. BROOKER,
G. (1970), Anal. Biochem. 42, 1108-1110. - 45. Steiner, A. L., KrPNIS, D. M., Utiger, R. \& PARKER, C. (1969), Proc. Nat. Acad. Sci. USA 64, 367. - 46. Steiner, A. L., PARKer, C. W. \& KipNIS, D. M. (1972), J. Biol. Chem. 247, 1106-1113. - 47. Yalow, R. S. \& Berson, A. S. (1960), J. Clin. Invest. 39, 1157. 48. Walsh, D. A., Perkins, J. P. \& Krebs, E. G. (1968), J. Biol. Chem. 243, 3763-3765. - 49. Langan, 'T. A. (1968), Science 162, 579. - 50. Mryamoto, E., Kuo, J. F. \& Greengard, P. (1969), Science 165, 63. - 51. Miyamoto, E., Kuo, J. F. \& Greengard, P. (1969), J. Biol. Chem. 244, 6395-6402. 52. Tao, M., Salas, M. L. \& Lipman, F. (1970), Proc. Nat. Acad. Sci. USA 67, 408. - 53. Yamamura, H., Kumon, A., Nishiyama, K., Takeda, M. \& Nrsinzuka, Y. (1971), Biochem. Biophys. Res. Commun. 45, 1560. - 54. ErlichmanN, J., Hirsch, A. H. \& Rosen, O. M. (1971), Proc. Nat. Acad. Sci. USA 68, 731. 55. Kuo, J. F. \& Greengard, P. (1970), J. Biol. Chem. 245, 2493-2498. - 56. KUo, J. F., Wyatt, G. R. \& Greengard, P. (1971), J. Biol. Chem. 246, 7159-7167. - 57. Kuo, J. F. \& Greengard, P. (1970), J. Biol. Chem. 245, 4067-4073. 58. Wastila, W. B., Stull, J. T., Mayer, S. E. \& Walsh, D. A. (1971), J. Biol. Chem. 246, 1996-2003. - 59. Butcher, F. R. (1971), Horm. Metab. Res. 3, 336-340. - 60. Kuo, J. F., LeE, T. P., Reyes, P. L., Walton, K. G., Donelly, T. E. jr. \& GreenGard, P. (1972), J. Biol. Chem. 247, 16-22. - 61. Walton, G. M. \& Garren, L. D. (1970), Biochemistry 9, 4223-4229. 62. Gilman, A. G. (1970), Proc. Nat. Acad. Sci. USA 67, 305-312. 63. Brown, B. L., Albano, J. D. M., Ekins, R. P., Sgiherzi, A. M. \& Tampion, W. (1971), Biochem. J. 121, 561-562. 64. Wombacher, $\dot{H}$. \& Körber, F. (1972), diese Z. 10, 260-266. 65. Weller, M., Rodnight, R. \& Carrera, D. (1972), Biochem. J. 129, 113-121. - 66. FisCh, H. U., PlisKa, V. \& SCHWyZER, R. (1972), Eur. J. Biochem. 30, 1-6. - 67. Cheung, W. Y. (1972), Biochem. Biophys. Res. Commun. 46, 99-105. - 68. O'DEA R. F., HaDdoX, M. K. \& GoldBerG, N. D. (1971), J. Biol. Chem. 246, 6183-6190. - 69. PAIN, J. N., Ponnter, R. H. \& WARD, W. F. (1972), J. Biol. Chem. 247, 6866-6872. - 70. Yamashita, K. \& Field, J. B. (1972), J. Biol. Chem. 247, 7062-7066. 71. Rubin, C. S., ERlichman, J. \& Rosen, O. M. (1972), J. Biol. Chem. 247, 36-44. - 72. Rodbard, D., Bridson, W. \& RAYFORD, P. L. (1969), J. Lab. Clin. Med. 74, 770. - 73. MurAd, F., Manganiello, V. \& Vaughan, M. (1971), Proc. Nat. Acad. Sci. USA 68, 736-739. - 74. Murad, F. \& Gilman, A. G. (1971), Biochim. Biophys. Acta 252, 397-400. - 75. GerbItz, K.-D. \& WIELAND, O., unveröffentlichte Beobachtungen. - 76. BUTLER, D. \& JoRd, S. (1972), Pflügers Arch. 331, 172-190. - 77. Cramer, H., persönliche Mitteilung. - 78. Chase, L. R. \& Aurbach, G. D. (1967), Proc. Nat. Acad. Sci. USA 58, 518. 79. Schultz, G., Jakobs, K. Н., Böhme, Е. \& Schultz, K. (1972), Eur. J. Biochem. 24, 520-529. - 80. GEORGE, W. J., Polson, J. B., O'Toole, A. G. \& Goldberg, N. D. (1970), Proc. Nat. Acad. Sci. USA 66, 398. - 81. Williams, R., WAlsh, S., Hepp, K. D. \& Ensinck, J. (1968), Metab. Clin. Exp. 17, 653. - 82. Marcus, R. J., Wilber, J. F. \& Aurbach, G. D. (1971), J. Clin. Endocrinol. Metab. 33, 537. - 83. Arduino, F., Ferrz, F. P. J. \& Rodriguez, J. (1966), J. Clin. Endocrinol. Metab. 26, 1325. - 84. Reforzo-Membrives, J., Moledo, L. I., Lanaro, A. E. \& Megtas, A. (1968), J. Clin. Endocrinol. Metab. 28, 332. - 85. Webster, W. \& BaIN, J. (1970), J. Clin. Endocrinol. Metab. 30, 215. - 86. Brooker, G. \& Frchman, M. P. (1971), Biochem. Biophys. Res. Commun. 42, 824. - 87. Dirzron, B. R., Paul, M. I. \& Pauk, G. L. (1970), Pharmacology 3, 25. 88. Palmer, G. C., Robison, G. A. \& Sulser, F. (1971), Biochem. Pharmacol. 20, 236. - 89. Kodama, T., Matsukado, Y., Suzuki, T., TANaka, S. \& Shrmizu, H. (1971), Biochim. Biophys. Acta 252, 165. - 90. Pule, J. \& McIliwain, H. (1972), Biochem. J. 126, 965. - 91. Lust, W. D., Passonenu, J. V. \& GoldberG, N. D. (1972), Abstract, Fed. Proc. 30. - 92. Hamradah, K., Holmes, H. \& Barkek, G. (1972), Proc. Biochem. Soc. - 93. MarX, J. L. (1972), Science 178, 1188. - 94. White, L. IW. (1972), 45. Ann. Meeting Centr. Soc. Clin. Res. - 95. Rama- 
CHANDRAN, J. (1971), Anal. Biochem. 43, 227-239. - 96. WhITE, A. A. \& ZENSER, T. V. (1971), Anal. Biochem. 41, 372-396. - 97. Krishna, G., WeIss, B. \& BRodie, B. B. (1968), J. Pharmacol. Exp. Ther. 163, 379-385. - 98. Roth, J., Prour, T. E., Goldfine, J. D., Wolfe, S. M., Muenzer, J., Grauner, L. E. \& Marcus, M. L. (1971), Ann. Intern. Med. 75, 607-621. 99. Guthrow, C. E., Allen, J. E. \& Rasmussen, H. (1972), J. Biol. Chem. 247, 8145-8153. - 100. SANBorn, B. M., Bhalla, R. C. \& Korenman, S. T. (1973), Endocrinol. 92, 494-499. -
101. Aurbach, G. D., Marcus, R., Winickofr, R. N., Epstein, E. H. \& NigrA, T. P. (1970), Metab. Clin. Exp. 19, 799-808. 102. Castagna, M. (1970), FEBS-Letters 8, 289-292. - 103. Kobata, A. (1961), J. Biochem. (Tokyo) 50, 275. - 104. Murad, F. \& PAK, C. Y.C., Abstract p. 583 in l. c. (37). - 105. Linarelli, L. G., Abstract p. 580 in 1. c. (37). - 106. BECK, N. P. DE RUBERTIS, F. R., Michelis, M. F., Fusco, R. D., Field, J. B. \& Davies, B. B. (1970), J. Lab. Clin. Med. 76, 1005.
' Dr. K.-D. Gerbitz Prof. Dr. O. Wieland D-8000 München 40 Kölner Platz 1 